\title{
Conservation de tissu testiculaire et matura- tion in vitro de la lignée germinale pour préservation du potentiel de reproduction avant traitement anticancéreux chez le garçon pré-pubère.
}

\author{
H. LEJEUNE, P. DURAND \\ Clinique Endocrinologique, Hôpital de l'Antiquaille et INSERM-INRA U418, Hôpital \\ Debrousse, LYON, France.
}

\section{RÉSUMÉ}

La cryoconservation des spermatozoïdes avant traitement chimio-radiothérapique stérilisant permet de préserver des possibilités de reproduction chez l'homme adulte. En l'absence de spermatozoïdes matures, des mesures de préservation du potentiel de reproduction n'ont pas pu, jusqu'ici, être envisagées chez l'enfant, alors même que le taux de guérison de certaines pathologies malignes est important à cet âge. Cette revue se propose de faire le point sur les avancées récentes qui ouvrent des perspectives de conservation et de maturation in vitro de cellules germinales masculines immatures qui pourraient être prélevées chez les garçons pré-pubères avant traitement chimio-radiothérapique.

Il a été montré que certaines étapes de la méiose ont pu être réalisées in vitro, avec obtention de spermatides à partir de spermatocytes de rats. Des spermatides rondes cryoconservées ont été utilisées pour féconder des ovocytes de souris et ont permis d'obtenir des souriceaux. Dans l'espèce humaine, des grossesses ont pu être obtenues à la suite de microinjections de spermatides rondes dans des cas d'azoospermie. Une grossesse a été rapportée en utilisant une spermatide ayant subi une cryoconservation. Ainsi le projet de prélèvement d'un fragment de parenchyme testiculaire avant traitement stérilisant, de maturation in vitro des spermatogonies jusqu'au stade de spermatide, de cryoconservation des spermatides, et de leur utilisation ultérieure pour microinjection intra-ovocytaire dans le but d'obtenir une grossesse, n'apparaît plus irréalisable, puisque chaque étape a pu être réalisée individuellement. La mise au point chez l'animal d'une telle procédure apparaît un préalable indispensable pour en étudier la faisabilité mais aussi l'innocuité avant le passage à une phase d'application clinique.

Mots-Clés : Chimiothérapie, radiothérapie, enfant, spermatogenèse in vitro, cryoconser-vation, spermatide.

L'intensification des traitements par radiothérapie et chimiothérapie permet d'augmenter la survie dans les maladies cancéreuses, notamment chez l'enfant. Il a ainsi pu être estimé que, dans la population des sujets âgés de 20 ans, $0,1 \%$ des sujets sont des survivants d'un cancer de l'enfance [25]. Ces traitements ont des effets secondaires, ils sont en particulier responsables d'altérations testiculaires et de stérilité. La cryoconservation des spermatozoïdes avant traitement chimio-radiothérapique stérilisant permet de préserver des pos- 
sibilités de reproduction chez l'homme adulte. En l'absence de spermatozoïdes matures, des mesures de préservation du potentiel de reproduction n'ont pas pu, jusqu'ici, être envisagées chez l'enfant, alors même que le taux de guérison est important à cet âge $(\approx 65-70 \%$ de guérison dans les leucémies aiguës).

Cette revue se propose de faire le point sur les avancées récentes qui ouvrent des perspectives de conservation et de maturation in vitro de cellules germinales masculines immatures qui pourraient être prélevées chez les garçons prépubères avant traitement chimio-radiothérapique et utilisées ultérieurement dans un but de procréation, après guérison de la maladie cancéreuse.

\section{LE TESTICULE PRÉ-PUBÈRE ET LA MISE EN PLACE DE LA SPERMATOGE- NÈSE}

Les études sur l'évolution des cellules germinales chez le garçon de la naissance à la puberté sont assez rares. Il apparaît néanmoins que le volume testiculaire augmente progressivement de 1,1 à $3 \mathrm{ml}$ et cela du fait de l'accroissement de la longueur des tubes séminifères [15]. Les tubes séminifêres sont constitués de cellules de Sertoli et de spermatogonies. Le nombre de spermatogonies visibles par section de tube séminifere varie au cours de l'enfance : il diminue légèrement de la naissance à l'âge de 3 ans puis augmente jusqu'à l'âge de 8 ans, diminue de nouveau entre 8 et 9 ans pour augmenter ensuite jusqu'à la puberté. On note, en particulier dans la période entre 3 et 8 ans, que certaines spermatogonies se localisent en position plus centrale dans le tube séminifêre et présentent des anomalies morphologiques (hypertrophie, 2 ou 3 noyaux) et des signes de dégénérescence [18]. Dans certains tubes séminifêres de certains garçons, on trouve quelques spermatocytes prenant le plus souvent un aspect dégénératif, et de rares spermatides qui dégénèrent sans qu'aucun spermatozoïde ne puisse être formé [16]. Il semble ainsi se produire pendant l'enfance une certaine activité de prolifération et de dégénérescence spermatogoniale, les tentatives de progression de la spermatogenèse échouent à cette période de la vie.
Ce n'est qu'à la puberté, sous l'effet des gonadotrophines que la spermatogenèse peut se dérouler jusqu'à son terme. Les gonadotrophines hypophysaires ont pour cellules cibles les cellules somatiques du testicule, cellules de Leydig pour la LH et cellules de Sertoli pour la FSH. Le développement de la spermatogenèse nécessite des interactions paracrines étroites entre cellules germinales et cellules somatiques du testicule. De nombreux facteurs paracrines assurant les communications entre cellules somatiques et cellules germinales ont été identifiés [9]. Nos connaissances des interactions entre cellules somatiques (cellules de Sertoli) et cellules germinales sont toutefois encore parcellaires, en particulier du fait des difficultés rencontrées jusqu'ici dans le développement de systèmes de cultures de cellules germinales à long terme.

\section{EFFETS DES TRAITEMENTS ANTI-CANCÉREUX PRÉ-PUBERTAIRES SUR LA SPERMATOGENĖSE.}

Les traitements anticancéreux pédiatriques provoquent des altérations de la lignée germinale pouvant être définitives, avec disparition complète des spermatogonies souches. La comparaison de l'intensité des effets secondaires des traitements anti-cancéreux en fonction de l'âge auquel ils sont appliqués, semble indiquer que les lésions de la lignée germinale sont moins intenses chez l'enfant que chez l'adulte [21], sans doute parce que la spermatogenèse n'est pas encore en activité chez l'enfant. Par contre, les lésions leydigiennes seraient plus intenses chez l'enfant.

On retrouve chez l'enfant comme chez l'adulte une toxicité différentielle selon les produits utilisés. Il est ainsi possible de privilégier, à efficacité égale, les protocoles thérapeutiques les moins agressifs sur la lignée germinale. Ainsi le remplacement du protocole MOPP par le protocole ABVD dans la maladie de Hodgkin, a permis une nette diminution des séquelles testiculaires [32]. Il n'est toutefois pas toujours possible d'éviter les traitements gonadotoxiques. L'intensification des traitements associés à une greffe de moelle, fait que la lignée germinale sera définitivement altérée chez bon nombre de patients qui vont survivre 
de leur maladie maligne sévère, au prix d'un traitement intensif.

Les tentatives de protection de la spermatogenèse pendant les traitements radio-chimiothérapiques par freinage gonadotrope ont donné des résultats paraissant prometteurs chez l'animal [14]. L'application en clinique humaine ne s'est malheureusement pas révélée réellement efficace chez l'homme adulte. Le principe de la mise au repos du testicule n'a pas de fondement chez l'enfant.

L'idée de cryoconserver les cellules germinales immatures obtenues chez l'enfant avant le début du traitement anti-cancéreux, comme on le fait pour les spermatozoïdes de l'homme adulte, nécessite que l'on puisse, ultérieurement, amener les cellules germinales immatures à un état de maturité compatible avec la "fécondation" d'un ovocyte et un développement embryonnaire normal, lorsque le sujet guéri aura un projet parental.

\section{AUTOGREFFES - DANGER DE RÉINTRODUCTION DE CELLULES MALIGNES}

Il a été montré que les cellules germinales mâles peuvent poursuivre leur différenciation [6] et donner naissance à des souriceaux [5], après avoir été isolées du testicule de souris, puis ré-injectées dans les tubes séminifères d'un autre animal génétiquement stérile ou rendu stérile par un cytotoxique (busulfan) préalable. Ceci a pu être réalisé aussi après congélation-décongélation des cellules germinales [3]. Ces expériences ont pu suggérer la possibilité de prélèvement d'un fragment de parenchyme testiculaire avant traitement stérilisant, cryoconservation de cellules germinales immatures en vue d'une "autogreffe" testiculaire ultérieure [4]. Des particularités d'espèce ont amené à préconiser, chez les primates, l'introduction des cellules germinales à transplanter par le rete testis [24]. Toutefois, la fréquence de la localisation testiculaire des cellules leucémiques rend l'auto-transplantation de cellules germinales immatures, prélevées avant traitement chimio-radiothérapique puis cryoconservées, potentiellement dangereuse en raison du risque de récidive testiculaire de la maladie maligne à partir des cellules leucémiques autologues initialement présentes dans le testicule et susceptibles de contaminer les cellules germinales.

Comme il a été montré chez les rongeurs qu'il n'y a pas de spécificité absolue d'espèce puisque des spermatozoïdes de rat ont pu être obtenus après transplantations de spermatogonies dans des testicules de souris immunodéficientes [7], il a été suggéré d'utiliser un animal hôte pour assurer la maturation des cellules germinales humaines de manière à n'utiliser que les spermatozoïdes pour une microinjection. Ceci pourrait permettre d'éviter le danger de récidive de la maladie maligne. On ne sait toutefois pas si cette possibilité de xéno-transplantation d'une espèce à l'autre est applicable en dehors des rongeurs. D'autre part, la possibilité de contamination des cellules germinales humaines par des rétrovirus animaux n'est pas exclue [23].

\section{MATURATION IN VTTRO DES CELLULES GERMINALES MÂLES}

Une procédure comportant la maturation in vitro des cellules germinales mâles et leur utilisation pour fécondation assistée après cryoconservation devrait permettre d'éviter le risque lié à la réintroduction dans le testicule de cellules malignes ou le recours à la xénotransplantation des cellules germinales.

Il a pu être démontré que certaines étapes de la spermatogenèse peuvent être réalisées in vitro avec obtention de spermatides à partir de spermatocytes de rat. Le passage de la méiose a pu être obtenu dans un système dissocié de coculture de cellules de Sertoli de rat immature (20 jours) et de spermatocytes isolés par élutriation à partir de testicules de rats adultes [33] ou dans un système de culture de tubes séminifêres mis en culture avant que la méiose ne se soit mise en place in vivo [10]. Dans l'espèce humaine, les possibilités de culture et d'études fonctionnelles des cellules testiculaires in vitro sont plus limitées que dans les modèles animaux. Il apparaît néanmoins possible de réaliser des cultures de cellules somatiques de testicules humains normaux à long terme, en gardant une activité fonctionnelle 
spécifique [13]. Dans des expériences à plus court terme, l'effet de la FSH et de la testostérone sur les cellules germinales humaines a été étudié à partir de biopsies testiculaires réalisées chez des patients présentant des obstructions des voies excrétrices [28, 29]. Ainsi, bien que les résultats soient encore parcellaires, la voie de la maturation in vitro des cellules germinales immature est ouverte. Cette démarche se justifie du fait que la possibilité de conception à partir de cellules germinales immatures a été démontrée.

\section{FÉCONDATION ASSISTÉE AVEC DES CELLULES GERMINALES IMMATURES}

Si le passage de la méiose in vitro a pu être démontré, il n'apparaît pas possible de permettre la phase de spermiogenèse in vitro à l'heure actuelle. Néanmoins, des cellules germinales immatures ont pu être utilisées à la place des spermatozoïdes pour obtenir une fécondation et un développement embryonnaire. Des spermatides rondes [12], éventuellement cryoconservées [26] ont pu être utilisées pour féconder, par microinjection ou électrofusion, des ovocytes de souris, ce qui a permis la naissance de souriceaux normaux. L'utilisation de cellules à des stades encore plus précoces a été tentée. Des spermatocytes secondaires [11] et même des spermatocytes primaires $[17,22]$ ont été utilisés chez la souris. La fin de la méiose mâle a pu s'accomplir dans l'ovocyte, à la faveur de la reprise de la méiose ovocytaire, avec expulsion du matériel génétique mâle excédentaire, sous forme de globule polaire supplémentaire, ce qui a permis d'observer un développement embryonnaire normal qui a pu aboutir à la naissance de souriceaux normaux.

Dans l'espèce humaine, des grossesses ont pu être obtenues à la suite de microinjections de spermatides rondes dans un ovocyte mature $[8$, $30,31]$. Contrairement aux travaux chez la souris, il s'agissait de cas pathologiques pour lesquels les spermatides rondes représentaient les cellules germinales les plus avancées dont on disposait le jour de la microinjection. Ces spermatides ont été obtenues par biopsie testiculaire ou dans l'éjaculat. Une grossesse a été rapportée en utilisant une spermatide ayant subi une cryoconservation [2]. Récemment il a été rapporté que, à partir de cellules testiculaires obtenues chez des sujets azoospermiques pour lesquels les cellules germinales les plus avancées observées étaient des spermatocytes, une culture de quelques jours, a permis la microinjection de spermatides et l'obtention de grossesses [27]. Des méthodes d'identification [1] et d'isolement des spermatides rondes à l'état vivant [34] ont été mises au point de manière à éviter que des ovocytes ne soient microinjectés par erreur avec un autre type de cellule.

Ainsi un projet de prélèvement d'un fragment de parenchyme testiculaire avant traitement stérilisant, maturation in vitro des cellules germinales jusqu'au stade de spermatide, cryoconservation des spermatides, et utilisation ultérieure pour microinjection intra-ovocytaire dans le but d'obtenir une grossesse mérite d'être pris en considération dans une perspective de préservation du potentiel de fertilité après traitement anti-cancéreux chez l'enfant puisque la plupart des étapes ont été réalisées individuellement.

\section{DIFFICULTÉS ET DANGERS}

Certains points ne sont pas encore résolus, et l'estimation des risques d'une telle procédure doit être envisagée de manière théorique mais aussi expérimentale.

Si les travaux résumés ci-dessus montrent le passage de la méiose in vitro, la phase d'entrée en méiose des spermatogonies n'a pas encore été démontrée de manière formelle in vitro. Le franchissement de cette étape correspond au passage de l'état pré-pubère à la spermatogenèse adulte. Il reste à déterminer dans quelles conditions les cellules de Sertoli vont être capables de promouvoir l'entrée en méiose des spermatogonies à partir du moment où elles sont soumises à une stimulation hormonale mimant la puberté.

Si on peut observer des signes de début de différenciation spécifiques des spermatides obtenues in vitro chez le rat $[10,19,33]$, toutefois le stade de spermatide ronde n'est pas dépassé et les possibilités de spermiogenèse in vitro apparaissent limitées. Les risques liés à l'utili- 
sation de spermatides rondes dans une perspective de procréation ont été largement discutés à la suite des essais initiaux réalisés dans l'espèce humaine avant même que la procédure n'ait été testée dans des modèles animaux.

Les interrogations portent sur

1) le stade auquel s'établit l'empreinte génomique ("imprinting") dans les cellules germinales au cours de la spermatogenèse,

2) le rôle protecteur des protéines nucléaires spécifiques des spermatides (protéines de transition, protamines),

3) le mécanisme d'activation de l'ovocyte dans les procédures de microinjection des spermatides,

4) le rôle du centriole masculin dans la polarisation de l'ovocyte et le développement embryonnaire. Il est possible que l'immaturité de ces phénomènes puisse être responsable $\mathrm{du}$ faible nombre de grossesses menées à terme à partir des spermatides rondes par rapport aux meilleurs résultats obtenus à l'aide des spermatides en voie d'élongation [20].

Il faut toutefois souligner que l'interprétation des résultats obtenus dans les cas pathologiques humains ne peut pas être extrapolée sans précaution au projet de cryoconservation avant traitement anticancéreux. La cause initiale de la perturbation de la spermatogenèse est différente, une pathologie souvent mal comprise à l'échelon moléculaire dans les azoospermies et un état pré-pubère chez des enfants devant subir un traitement anticancéreux. De même la cause du blocage de la spermiogenèse chez les sujets azoospermiques est vraisemblablement différente des difficultés de maintien des cultures des cellules testiculaires à très long terme, ce qui empêche la progression de la spermiogenèse in vitro.

La question plus générale du risque de favoriser la transmission d'une susceptibilité au cancer doit aussi être examinée avant de développer une procédure permettant à des sujets ayant eu un cancer pédiatrique d'avoir des enfants. Une étude récente portant sur 5847 enfants de 14652 sujets survivants d'un cancer pédiatrique (mais dont la fertilité a été préser- vée) montre que, mis à part les cancers relevant d'une transmission génétique bien établie comme le rétinoblastome, la fréquence des autres cancers n'est pas significativement accrue dans la descendance, alors même que les sujets (et leurs cellules germinales) ont subi le traitement du cancer.

Une mise au point préalable sur des modèles animaux apparaît indispensable en vue de préciser non seulement la faisabilité mais aussi l'innocuité de la procédure par l'étude d'un nombre significatif de produits de conception obtenus à la suite de la procédure complète. L'adaptation à l'espèce humaine devrait comporter des études préalables à partir de tissus testiculaires humains normaux comme ceux que l'on a pu prélever chez des sujets en état de mort cérébrale récente, lors des prélèvements d'organe pour transplantation. Ces études, qui ont été rendues pratiquement impossibles à la suite des dispositions de la loi bioéthique de 1994, pourront peut-être reprendre lorsque la loi bioéthique aura été rediscutée. Par ailleurs les procédures devront être adaptées en fonction des règles de sécurité sanitaire en évitant les risques de transmission de zoonose.

La toxicité gonadique des traitements anticancéreux étant variable et les protocoles thérapeutiques se modifiant, les indications de prélèvement de tissu testiculaire pour maturation in vitro, cryoconservation et conception assistée ultérieure doivent être précisées. Il s'agit en effet de préciser les cas pour lesquels la fonction de reproduction sera définitivement compromise par le traitement radio-chimiothérapique, alors que les chances de guérison laissent prévoir un désir de paternité ultérieure.

Le délai entre le traitement radio-chimiothérapique chez le garçon pré-pubère et le désir de paternité sera de plus de 10 ans. Ceci implique une cryoconservation des cellules germinales immatures d'assez longue durée. Le stade auquel les cellules germinales immatures subiront le moins d'altération lors du processus de congélation-décongélation reste à déterminer, la congélation pouvant porter sur les cellules germinales immatures au stade de spermatogonies avant maturation in vitro ou au stade de spermatides rondes après la méiose. 


\section{CONCLUSION}

Ainsi le projet de prélèvement d'un fragment de parenchyme testiculaire avant traitement stérilisant, la maturation in vitro des cellules germinales jusqu'au stade de spermatide, la cryoconservation des cellules germinales immatures, et l'utilisation ultérieure des spermatides pour microinjection intra-ovocytaire dans le but d'obtenir une grossesse n'apparaît plus irréalisable. La majorité des étapes a pu être réalisée individuellement. Une mise au point préalable chez l'animal et une adaptation des méthodes dans l'espèce humaine sont nécessaires pour établir non seulement la faisabilité mais aussi l'innocuité de la procédure avant de débuter la phase thérapeutique. Celle-ci sera envisagée dans les cas pour lesquels le pronostic de fertilité est clairement défavorable alors que le pronostic vital à long terme est suffisamment bon pour permettre un désir de paternité après guérison de la maladie cancéreuse.

\section{RÉFÉRENCES}

1. ANGELOPOULOS T., KREY L., MCCULLOUGH A., ADLER A., GRIFO J.A. : A simple and objective approach to identifying human round spermatids. Hum Reprod, 1997, 12 : 2208-2216.

2. ANTINORI S., VERSACI C., DANI G., ANTINORI M., SELMAN H.A. : Successful fertilization and pregnancy after injection of frozen-thawed round spermatids into human oocytes. Hum Reprod, 1997, 12 : 554-556.

3. AVARBOCK M.R., BRINSTER C.J., BRINSTER R.L.: Reconstitution of spermatogenesis from frozen spermatogonial stem cells. Nature Med, 1996, $2: 693-696$.

4. BAHADUR G., RALPH D. : Gonadal tissue cryopreservation in boys with paediatric cancers. Hum Reprod, 1999, 14 : 11-17.

5. BRINSTER R.L., AVARBOCK M.R. : Germline transmission of donor haplotype following spermatogonial transplantation. Proc Natl Acad Sci USA, 1994, 91 : 11303-11307.

6. BRINSTER R.L., ZIMMERMANN J.W. : Spermatogenesis following male germ-cell transplantation. Proc Natl Acad Sci USA, 1994, $91:$ 11298-11302.

7. CLOUTHIER D.E., AVARBOCK M.R., MAIKA S.D., HAMMER R.E., BRINSTER R.L. : Rat spermatogenesis in mouse testis. Nature, 1996, $381: 418-421$.

8. FISHEL S., GREEN S., BISHOP M., et al. : Pregnancy after intracytoplasmic injection of spermatid. Lancet, 1995, $345: 1641-1642$.
9. GNESSI L., FABBRI A., SPERA G. : Gonadal peptides as mediators of development and functional control of the testis: An integrated system with hormones and local environment. Endocrine Rev, 1997, $18: 541-609$.

10. HUE D., STAUB C., PERRARDSAPORI M.H., et al. : Meiotic differentiation of germinal cells in three-week cultures of whole cell population from rat seminiferous tubules. Biol Reprod, 1998, 59 : 379-387.

11. KIMURA Y., YANAGIMACHI R. : Development of normal mice from oocytes injected with secondary spermatocyte nuclei. Biol Reprod, 1995, 53 : 855-862.

12. KIMURA Y., YANAGIMACHI R. : Mouse oocytes injected with testicular spermatozoa or round spermatids can develop into normal offspring. Development, 1995, $121: 2397-2405$.

13. LEJEUNE H., SANCHEZ P., SAEZ J.M. : Enhancement of long-term testosterone secretion and steroidogenic enzyme expression in human Leydig cells by co-culture with human Sertoli cell-enriched preparations. Int J Androl, 1998, 21 : 129-140.

14. MEISTRICH M.L. : Hormonal stimulation of the recovery of spermatogenesis following chemo- or radiotherapy - Review article. Apmis, 1998, $106:$ 37-45.

15. MULLER J., SKAKKEBAEK N.E. : Quantification of germ cells and seminiferous tubules by stereological examination of testicles from 50 boys who suffered from sudden death. Int J Androl, 1983, 6 : 143-156.

16. NISTAL M., PANIAGUA R. : Occurrence of primary spermatocytes in the infant and child testis. Andrologia, 1984, 16 : 532-536.

17. OGURA A., WAKAYAMA T., SUZUKI O., et al. : Chromosomes of mouse primary spermatocytes undergo meiotic divisions after incorporation into homologous immature oocytes. Zygote, 1997, 5 : 177182.

18. PANIAGUA R., NISTAL M. : Morphological and histometric study of human spermatogonia from birth to the onset of puberty. J Anat, 1984, $139: 535-552$.

19. PERRARD-SAPORI M.H., VIGIER M., HUE D., et al.: Spermatogénèse murine in vitro : étude cytologique de l'étape méiotique. Contracept Fertil Sex, 1997, 25 : 556-564.

20. PRAPAS Y., CHATZIPARASIDOU A., VANDERZWALMEN P., et al. : Spermatid injection: reconsidering spermatid injection. Hum Reprod, 1999, 14 : 2186-2188.

21. RIVKEES S.A., CRAWFORD J.D. : The relationship of gonadal activity and chemotherapy-induced gonadal damage. JAMA, 1988, $259: 2123-2125$.

22. SASAGAWA I., KURETAKE S., EPPIG L.J., YANAGIMACHI R. : Mouse primary spermatocytes can complete two meiotic divisions within the oocyte cytoplasm. Biol Reprod, 1998, 58 : 248-254. 
23. SCHLATT S. : Prospects and problems for germ cell transplantation in the male. Int J Androl, 1999, 22 : 13-18.

24. SCHLATT S., ROSIEPEN G., WEINBAUER G.F., et al. : Germ cell transfer into rat, bovine, monkey and human testes. Hum Reprod, 1999, 14 : 144-150.

25. SCHWARTZ C.L. : Long-term survivors of childhood cancer: the late effects of therapy. Oncologist, 1999, 4 : 45-54.

26. TANEMURA K., WAKAYAMA T., KURAMOTO K., et al. : Birth of normal young by microinsemination with frozen-thawed round spermatids collected from aged azoospermic mice. Lab Anim Sci, 1997, 47 : 203-204.

27. TESARIK J., BAHCECI M., OZCAN C., GRECO E., MENDOZA C. : Restoration of fertility by in-vitro spermatogenesis. Lancet, 1999, $353: 555-556$.

28. TESARIK J., GRECO E., RIENZI L., et al. : Differentiation of spermatogenic cells during in-vitro culture of testicular biopsy samples from patients with obstructive azoospermia: effect of recombinant follicle stimulating hormone. Hum Reprod, 1998, 13 : 2772-2781.

29. TESARIK J., GUIDO P., MENDOZA C., GRECO E. : Human spermatogenesis in vitro: Respective effects of follicle-stimulating hormone and testosterone on meiosis, spermiogenesis, and sertoli cell apoptosis. J Clin Endocrinol Metab, 1998, 83 : 4467-4473.

30. TESARIK J.N., MENDOZA C., TESTART J. : Viable embryos from injection of round spermatids into oocytes. N Engl J Med, 1995, 333 : 525.

31. VANDERZWALMEN P., LEJEUNE B., NIJS M., et al. : Fertilization of an oocyte microinseminated with a spermatid in an in-vitro fertilization programme. Hum Reprod, 1995, $10:$ 502-503.

32. VIVIANI S., SANTORO A., RAGNI G., et al. : Gonadal toxicity after combination chemotherapy for Hodgkin's disease. Comparative results of MOPP vs ABVD. Eur J Cancer Clin Oncol, 1985, 21 : 601-605.

33. WEISS M., VIGIER M., HUE D., et al. : Pre- and postmeiotic expression of male germ cell-specific genes throughout 2-week cocultures of rat germinal and Sertoli cells. Biol Reprod, 1997, 57 : 68-76.

34. ZIYYAT A., LASSALLE B., TESTART J., et al. : Flow cytometry isolation and reverse transcriptase polymerase chain reaction characterization of human round spermatids in infertile patients. Hum Reprod, 1999, $14: 379-387$.
ABSTRACT

Conservation of testicular tissues and in vitro maturation of male germ cells for preservation of reproductive potential before cancer treatment in prepubertal boy.

\section{H. LEJEUNE, P. DURAND}

Sperm auto-conservation before chimioradiotherapy allows preservation of future reproductive possibilities in case of malignancy in young adult male. Because of the lack of mature spermatozoa, such possibilities cannot be offered for boys before puberty, even though the rate of cure of childhood malignancies is high. This paper reviewed recent advances in reproductive technology, which open the field of withdrawal of immature germ cells in prepubertal boys for in vitro maturation and cryopreservation for future paternity. It has been shown that the main steps of male meiosis have been driven in vitro, allowing to obtain round spermatids from pachytene spermatocytes in the rat. In mice, cryopreserved round spermatids have been used for oocyte fertilization and gave rise to normal living pups. In humans pregnancies and living babies have been reported after microinjection of round spermatids in cases of azoospermia. One pregnancy has been obtained with a cryopreserved spermatid. Thus the project of withdrawal of testicular tissues before sterilizing treatment, in vitro maturation of spermatogonia into round spermatids and cryopreservation of immature germ cells for future use for assisted fertilization does not seem unrealistic since each step has been done individually. However developing animal models is necessary to study not only the efficiency of the whole procedure but also to check its harmlessness before clinical trials.

Key words : Chimiotherapy, radiotherapy, chilhood cancer, in vitro spermatogenesis, cryopreservation, spermatids. 